\title{
A rare cause of acute abdominal distention: opening of the pancreatic duct into hydatic cyst
}

\author{
Mehmet Agin M.D. ${ }^{a}$, Gokhan Tumgor M.D. ${ }^{a}$, Suzan İcil M.D. ${ }^{b}$, Serdar İskit M.D. ${ }^{c}$, Derya Alabaz M.D. ${ }^{d}$ and \\ Huseyin T. Ballı M.D. ${ }^{e}$
}

\begin{abstract}
Introduction. Hydatid cyst, which is caused by Echinococcus granulosus, is mostly seen in the liver and lungs although it may also rarely be found in any organ or soft tissue. This study presents an interesting case of pancreatic hydatid cyst in which the pancreatic duct opened into this cyst.

Case report. A 10-year-old boy presented to our clinic with significant abdominal distension and pain in the epigastric region which had started 10 days previously. Serum amylase level was 3709U/Land hemagglutination inhibition for hydatid disease was 1/160. At abdominal computed tomography, two separate lesions and ascites were determined, a CE2 hydatid cyst in the region of the tail of the pancreas and a CE1 hydatid cyst in the left lobe of the liver. Percutaneous drainage was applied to the cyst in the pancreatic tail, and the patient was started on albendazole. The drainage catheter was removed, and the patient has since been followed-up on an outpatient basis with no complications.

Key words: Hydatid cyst, pancreas, Echinococcus granulosus, pancreatitis, amylase.
\end{abstract}

http:/ /dx.doi.org/10.5546/aap.2016.eng.e346

\section{INTRODUCTION}

Hydatid cyst is a worldwide zoonotic infection that affects human and animal health, and it is the cause of significant economic loss for the agricultural sectors and public health systems in the endemic area. ${ }^{1}$ It is a frequently observed parasitosis in countries with widespread agriculture and stockbreeding sectors and insufficient levels of preventive medicine. ${ }^{2,3}$

a. Department of Pediatric Gastroenterology, Hepatology and Nutrition.

b. Department of Pediatric.

c. Department of Pediatric surgery.

d. Department of Pediatric infectious Diseases.

e. Department of Radiology.

Cukurova University Medical Faculty. Adana, Turkey.

E-mail Address:

Mehmet Agin M.D.: drmehmet47@yahoo.com

Funding: None.

Conflict of interest: None.

Received: $12-23-2015$

Accepted 2-25-2016
Hydatid cyst, which is caused by E. granulosus, is mostly seen in the liver and lungs, although it may also rarely be found in any organ or soft tissue. This paper presents an interesting case of pancreatic hydatid cyst and of the pancreatic channel opened onto this.

\section{Case report}

A 10-year-old boy with no previous symptoms presented to our clinic with abdominal pain and distension. These symptoms had started 10 days previously. The distension worsened rapidly, and he was transferred to our clinic. Physical examination findings were weight: $24 \mathrm{~kg}$ (10-25 percentile), height: $137 \mathrm{~cm}$ (90 percentile), body temperature: $37^{\circ} \mathrm{C}$, respiratory rate: $24 / \mathrm{min}$, pulse: 88/min and TA: 100/70 mmHg. The abdomen was distended and causing respiratory problems. The epigastrum and abdomen was sensitive to palpation. Other system examinations and complete blood count were normal. Serum amylase level was $3709 \mathrm{U} / \mathrm{L}$, urine amylase $1641 \mathrm{U} / \mathrm{L}$ and serum lipase $323 \mathrm{U} / \mathrm{L}$, and other biochemical parameters were normal (Table 1). Ascites was determined at abdominal ultrasonography (USG), and lobulated cystic lesion was determined in the left quadrant of the abdomen. Two separate lesions and ascites were determined at abdominal computed tomography (CT); a CE2 hydatid cyst in the pancreatic tail region $(14.5 \times 10 \mathrm{~cm})$ and a CE1 hydatid cyst in the left lobe of the liver ( $2 \mathrm{~cm}$ in diameter) (Figure 1) and ascites in the perihepatic and perisplenic region (Figure 2). hemagglutination inhibition for hydatid disease (HAI) value was 1/160. CT of the brain and thorax performed to scan for hydatid cyst was normal. Percutaneous drainage was applied to the cyst in the pancreatic tail, and the patient was started on albendazole. A dosage of $15 \mathrm{mg} / \mathrm{kg} /$ day of albendazole was starded preoperatively when the diagnosis of pancreatic hydatid disease was established and continued postoperatively for one month. Following drainage, the abdominal distension improved. The amylase level in drainage fluid was $336502 \mathrm{U} / \mathrm{L}$. This high amylase value in the drainage fluid suggested that the pancreatic duct 
had opened into the pre-existing hydatid cyst and caused it to grow rapidly. Following percutaneous drainage and albendazole therapy an apparent recovery was observed in laboratory and clinic findings. Control abdominal magnetic resonance imaging (MRI) revealed that the pancreatic cyst had decreased in size to $3.6 \mathrm{~cm}$ (Figure 3). The drainage catheter was removed, and the patient was transferred to the clinic for follow-up. The patient is currently in the first year of follow-up and no complications have arisen to date.

\section{DISCUSSION}

The annual surgical cases of hydatid cyst in Turkey are reported to be 0,9-6,6 per 100000 population; ${ }^{4}$ however, epidemiologic studies have reported higher prevalence rates 150-6884 per 100000 population., Although hydatid cysts can localize to almost any tissue or organ of the human body, the liver (50-77\%), lung (15$47 \%)$, spleen $(0.5-8 \%)$, and kidney $(2-4 \%)$ are the most commonly involved organs. ${ }^{7,8}$ While the reported incidences of pancreatic hydatid cyst

TABLE 1: Hemogram and biochemical parameters of the patient

\begin{tabular}{lccc}
\hline & On admission & At discharge & Normal range \\
\hline Leucocyte $\left(\mathrm{mm}^{3}\right)$ & 8700 & 5100 & $4500-13500$ \\
Hemoglobin $(\mathrm{gr} / \mathrm{dl})$ & 12 & 11 & $11,5-15,5$ \\
Thrombocytes $\left(\mathrm{mm}^{3}\right)$ & 603000 & 415000 & $150000-400000$ \\
Serum amylase (U/L) & 3709 & 120 & $30-100$ \\
Urine amylase (U/L) & 1641 & 416 & $400-600$ \\
Cyst fluid amylase (U/L) & 336502 & 125 & $30-100$ \\
Serum lipase (U/L) & 323 & 48 & $3-32$ \\
Total protein (g/dl) & 5,4 & 5,6 & $6,4-8,1$ \\
Albumin (g/dl) & 2,7 & 3,4 & $4-5,3$ \\
AST (U/L) & 87 & 44 & $5-45$ \\
ALT (U/L) & 81 & 37 & $5-45$ \\
GGT (U/L) & 8 & 8 & $5-32$ \\
Total bilirubin (mg/dL) & 0,2 & 0,2 & $0,3-2$ \\
Direct bilirubin (mg/dL) & 0,7 & 0,1 & $0,1-0,2$ \\
\hline
\end{tabular}

AST: Aspartate aminotransferase, ALT: Alanine aminotransferase, GGT: Gamma-glutamyltransferase

FIGURE 1: The cystic lesion which contains membranes in the posterior and in the size of $130 \times 110 \mathrm{~mm}$ located in the tail of the pancreas

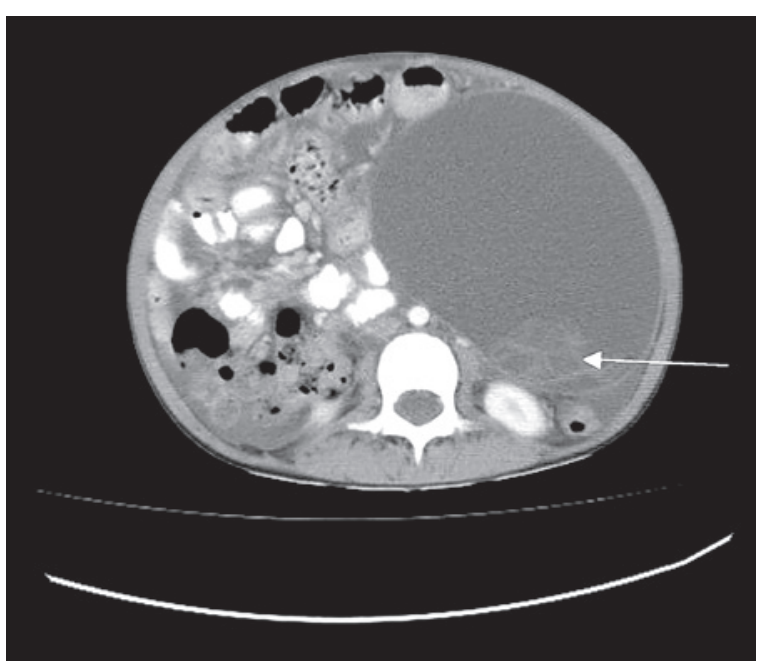

FIGURE 2: Ascites in the perihepatic and perisplenic region

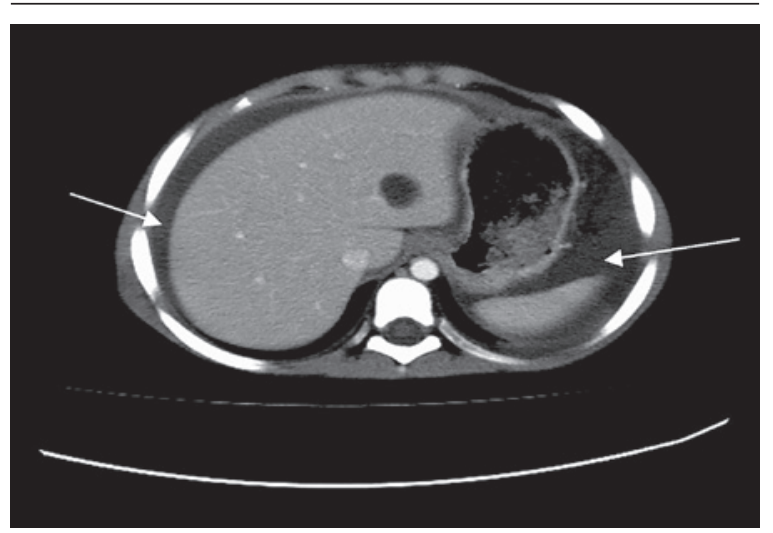


(PHC) have varied in different studies, the rates are consistently below $1 \%$. PHC may develop as a primary (involving the pancreas only) or secondary (with multiple organ involvement) disease. ${ }^{9}$ In pancreatic involvement, spread occurs locally through peripancreatic lymphocytic invasion, the pancreaticobiliary ductal system and hematogenous spread. Cyst location in pancreas was reported for 54 patients, where in the cyst was localized to the pancreatic head in 21 (38.8\%), the pancreatic tail in $18(33.3 \%)$, the pancreatic body and tail in $8(14.8 \%)$, the pancreatic body in $5(9.2 \%)$, the pancreatic head and body in 1 , and the pancreatic neck in $1 .{ }^{10}$ Since hydatid cysts grow slowly, a considerable portion of affected patients may remain asymptomatic for years. In symptomatic patients, however, the symptoms are varied and depend on location, size, and position relative to neighboring organs. ${ }^{11}$ The most serious complications in PHC disease are jaundice, cholangitis, and pancreatitis, all of which can develop as a result of fistulization of the cyst content into pancreato-biliary ducts or external compression of those ducts by the cyst. ${ }^{11}$ In symptomatic cases, mass and pain in the abdomen, nausea and vomiting may be seen.

Hepatitis, cholangitis, pancreatitis, pancreatic abscess and pancreatic fistula may occur, depending on the pressure caused by the cyst on the pancreatic duct or erosion of the cyst into the duct. ${ }^{12,13}$ Diagnosis of PHC is very difficult since the symptoms and findings are very similar to those of other pancreatic cyst diseases. Clinical and radiological imaging techniques and serological

FIGURE 3: The cyst hidatid concordant lesion in the size of $39 \times 36 \mathrm{~mm}$ which contains germinative membrane and is located in Axiel T2 weighted sequence in the pancreatic tail

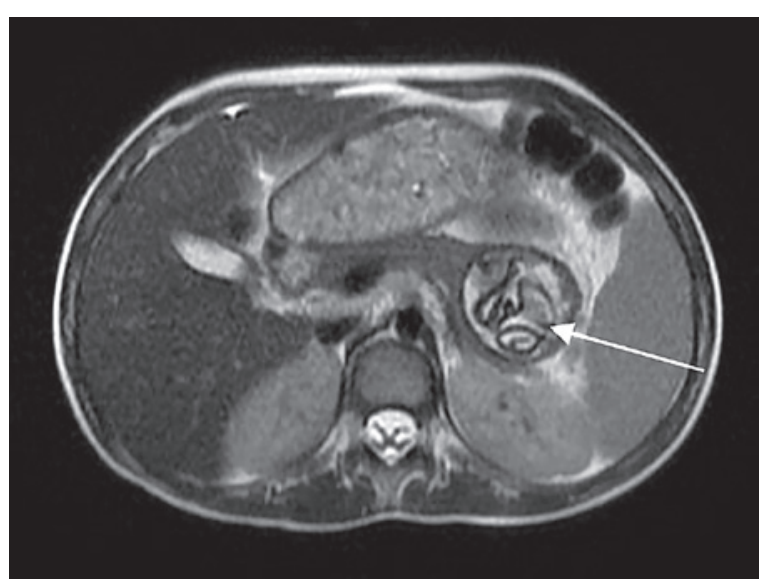

tests should be used. Since they are inexpensive and easy to apply, serological tests are used in the diagnosis and follow-up of the disease in countries where hydatid cyst is endemic. Reports in the literature describe sensitivities of $51-60,9 \%$ for HAI. ${ }^{14,15}$

Since there is no standard protocol for the treatment of hydatid cyst, the approach adopted should depend on the clinical, radiological and serological test results. ${ }^{2}$ Treatment of hydatid cyst can be surgical or medical with puncture aspiration - injection - reaspiration (PAIR). Since the HAI test is available in our hospital and also gives quick results, it was applied in our case and the results were significantly high. Amylase levels in serum, urine and drainage fluid were high. Since the case involved a very large pancreatic cyst and the amylase levels in drainage fluid were very high, we considered that the pancreatic duct was open into the cyst. Ascites was not a significant finding, but more probably a small mechanical spill due to the high cyst pressure, which may itself be confused clinically with ascites. It is therefore important to perform ultrasonography before deciding on abdominal puncture, as we performed in order to prevent hydatid dissemination.

Although high levels of amylase and lipase, presence of abdominal pain and a large cyst in the pancreas primarily suggest pancreatitis, abdominal pain is explained by the distension of the cyst and the same pressure on the abdomen. Increased serum amylase may be related to the discharge into the cyst (cystic membranes are semi-permeable especially when they are young and not thickened or calcified).

The patient was started on albendazole, and PAIR was applied. At follow-up, we observed a decrease in the size of the and also improvement in clinical and laboratory parameters. The patient has since been followed-up on an outpatient basis with no complications.

In conclusion, hydatid cyst, which is endemic in Turkey and its region, can progress asymptomatically or may exhibit symptoms and findings depending on organ involvement and location. Although PHC is rare, it should be primarily considered in the differential diagnosis of pancreatic cysts, especially in endemic regions. This case is described since it was complicated, with the patient presenting with ascites and epigastric, abdominal pain due to a rarely located large hydatid cyst in the pancreas and with the pancreatic duct opening, and with successful treated with PAIR and albendazole. 


\section{REFERENCES}

1. Rojo-Vazquez FA, Pardo-Lledias J, Francos-Von Hunefeld M,Cordero-Sánchez M, etal.Cystic echinococcosis in Spain: current situation and relevance for other endemic areas in Europe. PLoS Negl Trop Dis 2011;5(1):e893.

2. Sayek I, Tirnaksiz MB, Dogan R. Cystic hydatid disease: current trends in diagnosis and management. Surg Today 2004;34(12):987-96.

3. Biava MF, Dao A, Fortier B. Laboratory diagnosis of cystic hydatic disease. Word J Surg 2001;25(1):10-4.

4. Altintas N. Past to present: echinococcosis in Turkey. Acta Trop 2003;85(2):105-12.

5. Ok UZ, Ozkol M, Kilimcioğlu AA, Dinç G, et al. Provincebased study using sampling method to investigate the prevalence of cystic echinococcosis among primary school children in Manisa, Turkey. Acta Trop 2007;103(2):116-22.

6. Altintas N, Yazar S, Yolasigmaz A, Akisü C, et al. A seroepidemiological study of cystic echinococcosis in zmir and its surroundings area. Helminthologia 1999;36(1):19-23.

7. Eris C, Akbulut S, Yildiz MK, Abuoglu H, et al. Surgical approach to splenic hydatid cyst: single center experience. Int Surg 2013;98(4):346-53.
8. Yarlagadda P, Yenigalla BM,Penmethsa U,Myneni RB. Primary pancreatic echinococcosis. Trop Parasitol 2013;3(2):151-4.

9. Patil DS, Jadhav KV, Ahire PP, Patil SR, et al. Pancreatic hydatid presenting as an intercostal hernia. IJMAS 2013;2(3):255-8.

10. Akbulut S, Yavuz R, Sogutcu N, Kaya B, et al. Hydatid cyst of the pancreas: Report of an undiagnosed case of pancreatic hydatid cyst and brief literature review. World J Gastrointest Surg 2014;6(10):190-200.

11. Trigui A, Rejab H, Guirat A, Mizouni A, et al. Hydatid cyst of the pancreas About 12 cases. Ann Ital Chir 2013;84(2):165-70.

12. Wong S, Braghirolli-Neto O, Min Zu, Buckels J, Mirza D. Hydatid liver disease as a cause of recurrent pancreatic head. J R Coll Surg Edin 1999;44(6):407-9.

13. Barrera MC, Villanua J, Barrena JF, Nogues A. Pancreatic Hydatid disease. Pediatr Radiol 1995;25(Suppl 1):S169-70.

14. Gonlugur U, Ozcelik S, Gonlugur TE, Arici S, et al. The retrospective annual surgical incidence of cystic echinococcosis in Sivas, Turkey. Zoonoses Public Health 2009;56(5):209-14.

15. Wuestenberg J, Gruener B, Oeztuerk S, Mason RA, et al. Diagnostics in cystic echinococcosis: serology versus ultrasonography. Turk J Gastroenterol 2014;25(4):398-404. 